\title{
MARKETING DE UNIVERSIDADES, ¿OFERTA EDUCATIVA O IMAGEN?
}

UNIVERSITY MARKETING, EDUCATIONAL OFFER OR IMAGE?

\author{
Karen Giselle Acosta Ruiz*, Manuela Camacho Gómez**, \\ Julio Alejandro Zapata Cortes***
}

*Licenciada en Mercadotecnia. Universidad Juárez Autónoma de Tabasco.

**Doctora en Educación Internacional. Profesora Investigadora de la División Académica de Ciencias Económico Administrativas. Universidad Juárez Autónoma de Tabasco. Email: manuela.camacho@dacea.ujat.mx

***Licenciado en Ciencias de la Educación. Universidad Juárez Autónoma de Tabasco.

Dirección para recibir correspondencia: acostkgiselle@gmail.com 
MARKETING DE UNIVERSIDADES, ¿OFERTA EDUCATIVA O IMAGEN?

\section{RESUMEN}

OBJETIVO: Conocer la percepción que se tiene sobre el marketing en las universidades y su relevancia en la imagen institucional.

MATERIAL Y MÉTODO: La investigación se realizó con el método cualitativo fenomenológico, utilizando la técnica de sondeo flash, donde los informantes fueron estudiantes de pregrado de dos universidades: una pública y una privada.

RESULTADOS: Los principales resultados muestran una tendencia de los jóvenes para elegir instituciones con una imagen institucional reconocida socialmente, oferta de servicios educativos diferenciados, calidad y prestigio.

CONCLUSIONES: La percepción que los alumnos tienen de marketing universitario y de imagen institucional de sus universidades (pública y privada), lo confirman con el producto; es decir, con la oferta de servicios educativos y la calidad educativa que les ofrecen; además valoran la existencia de los planes estratégicos y el prestigio que estas han obtenido a lo largo del tiempo.

Para los estudiantes de la universidad privada, la imagen institucional se basa en la responsabilidad social universitaria y la oferta de servicios educativos, mientras que para los estudiantes de la universidad pública, la refieren como prestigio y reconocimiento social y destacan la calidad educativa de su institución.

PALABRAS CLAVE: Marketing universitario. Calidad educativa. Imagen institucional.

\section{ABSTRACT}

OBJECTIVE: The objective of this article was to know the perception of marketing in universities and its relevance in the institutional image.

MATERIAL AND METHOD: The research was carried out using the phenomenological qualitative method and the flash survey technique, where the informants were undergraduate students from two universities: one public and one private. 
MARKETING DE UNIVERSIDADES, ¿OFERTA EDUCATIVA O IMAGEN?

RESULTS: The main results show a tendency of young people to choose institutions with a socially recognized institutional image, offer of differentiated educational services, quality and prestige.

CONCLUSIONS: The perception that students have of university marketing and institutional image of their universities (public and private), is confirmed with the product, that is, with the offer of educational services and the educational quality that are offered to them; besides,, they also value the existence of strategic plans and the prestige that these institutions have obtained over time.

For the students of the private university, the institutional image is based on the university social responsibility and the offer of educational services, while for the students of the public university, it is based on the prestige and social recognition and they emphasize the educational quality of their institution.

KEYWORDS: University marketing. Educational quality. Institutional image.

\section{INTRODUCCIÓN}

La enseñanza como parte integrante de los 12 renglones de servicios incluidos en el Acuerdo General de Comercio de Servicios (AGCS) de la Organización Mundial del Comercio (OMC), contempla todos los niveles de educación, desde preescolar hasta la universidad. Por lo tanto, todas participan en los mercados educativos y en consecuencia, compiten con otras organizaciones. Así, el contexto comercial, ha llevado a que las instituciones públicas y privadas tradicionales pretendan expandir la oferta de sus servicios para aprovechar las oportunidades que les representan otros segmentos de mercado.

Los entornos competitivos de las Instituciones de Educación Superior (IES) se han transformado a partir de la diversificación de oferta privada y la reducción progresiva de accesos a la universidad, hecho que las ha condicionado a la competencia comercial que, antes sólo ejercían las IES con fines de lucro (Camacho, 2015). Con estas perspectivas de negocios, las universidades han aceptado paulatinamente el uso de estrategias de marketing, particularmente buscando dos metas: convencer a sus potenciales estudiantes (clientes) de la atractividad de su oferta educativa (producto) y del prestigio de la institución a partir del uso de 
MARKETING DE UNIVERSIDADES, ¿OFERTA EDUCATIVA O IMAGEN?

medios masivos y digitales (publicidad) para lograr con ello, el reconocimiento público que las avale (imagen corporativa).

El marketing universitario como tal ha sido cuestionado, ya que se concibe a la universidad como un ente social y no comercial. Sin embargo, algunos estudiosos le han dado su justa dimensión y aluden a las ventajas estratégicas que genera su aplicación (Tristá, 2002; Fayos, Gonzalez-Gallarza, Servera y Arteaga, 2011; Wörner y Santander, 2012, de la Fuente, Marzo y Reyes, 2010, Camacho, Ancona y García Muñoz, 2010 y Camacho, 2015).

Atendiendo esta dinámica de mercados de las universidades, el presente artículo tiene como propósito dar a conocer la percepción de los estudiantes acerca de los componentes académicos (oferta de servicios educativos) y el posicionamiento de la universidad a partir de la imagen institucional (imagen corporativa), el proyecto en los diferentes medios que utiliza. Con ello, se pretende añadir valor al estudio del marketing universitario en América Latina.

\section{REVISIÓN LITERARIA}

El marketing es una función estratégica de las organizaciones, su principal aporte consiste en dar a conocer un producto o servicio y convencer a mercados específicos sobre las particularidades de lo que se ofrece. En el marketing universitario, el uso de estas estrategias permite difundir la oferta educativa y atraer o captar a sus consumidores o clientes, quienes una vez egresados serán parte de la imagen corporativa de su institución. Así, es relevante mencionar que el marketing universitario tiene sus bases en el marketing de servicios, asumidas como actividades económicas que generalmente se basan en tiempo, valuado por los resultados esperados (Lovelock y Wirtz, 2009). Las tendencias en América Latina se encuentran en los trabajos de Tristá (2002) que sugiere la necesidad del marketing en las IES, estima que este tipo de marketing debe atender al menos cinco estrategias: académica, mercado objetivo, posicionamiento, competitividad y oportunidad (de mercado). Fayos, Gonzalez-Gallarza, Servera y Arteaga (2011), por su parte, refieren al marketing universitario como estratégico; aludiendo al servicio de formación universitaria como elemento fundamental. Worner y Santander (2012), dan a conocer la forma en que se lleva a cabo el marketing universitario en Chile y la relevancia de la inversión publicitaria para lograr una buena imagen institucional. De la Fuente, Marzo y Reyes (2010), revisan la satisfacción universitaria y afirman que existe un incremento de expectativas de la sociedad hacia las universidades lo que les ha requerido mejorar la calidad de la enseñanza universitaria para satisfacer a sus múltiples 
MARKETING DE UNIVERSIDADES, ¿OFERTA EDUCATIVA O IMAGEN?

clientes. Finalmente, en este breve recuento, Camacho, (2015) explica que el marketing universitario tuvo su aceptación a partir de la puesta en marcha el AGCS de la OMC, donde se incluye a la enseñanza como bien comercial.

En este contexto las estrategias publicitarias y promocionales del marketing universitario tienden a favorecer la imagen de la institución, colocándola como una marca. La identidad e imagen es definida como la cualidad de ésta como institución; la cual abarca elementos de gestión y de servicio; de sus atributos de solvencia, potencia, organización y eficacia (Costa, 1993). Es decir, la imagen corporativa de las universidades descansa en las estrategias de difusión, como si fuera una empresa. Por ello, se dirigen parte de los esfuerzos universitarios al diseño y oferta de servicios que de continuo se comercializan, y ha conducido a las universidades para que se desenvuelvan en un ambiente de alta competencia y competitividad, donde cohabitan o compiten con proveedores transnacionales, universidades de marca, universidades corporativas y consorcios que se dedican al comercio de servicios educativos (Camacho, 2011). La creación de universidades privadas ha sido uno de los principales factores que ha incrementado la competencia (Doña, 2014).

\section{MATERIAL Y MÉTODO}

El método que se utilizó en esta investigación fue el cualitativo con un enfoque de estudio de caso (Martínez, 2006). Los sujetos de estudio fueron 200 estudiantes de pre grado de dos universidades: una pública y una privada. Se decidió aplicar 100 en cada institución, con jóvenes del género femenino y masculino. Se tomó como base el estudio realizado por Camacho et al (2010) aplicado a estudiantes, profesores y administrativos de 11 universidades, encontraron que los estudiantes elegían a la institución donde se formarían profesionalmente, obedeciendo a cuatro factores: oferta de servicios educativos, imagen institucional, prestigio y precio.

Las técnicas de recolección de información fueron la revisión documental (impresa y digital), la observación participante y el sondeo flash (Arroba, 2000). El diseño de instrumentos fue de elaboración propia, orientado a conocer la percepción de imagen institucional y la influencia del marketing universitario. El análisis fue manual, apoyado con un benchmarking básico. 


\section{HALLAZGOS}

Como resultado de la investigación cualitativa se eligieron intencionalmente dos universidades, para conocer la percepción que tienen los estudiantes de pre grado sobre el marketing en las universidades y su influencia en la imagen institucional. En la observación participante, se pudo apreciar que los sujetos estudiados se sentían a gusto en sus respectivas universidades, disfrutando de la infraestructura y de los servicios que estas ofrecen.

El sondeo flash fue aplicado en la Universidad Juárez Autónoma de Tabasco (UJAT) (universidad pública) y, el Instituto Universitario de Puebla (IUP) (universidad privada), se eligieron intencionalmente estas organizaciones por su representatividad en el sector y en la entidad (ver tabla 1).

\section{Tabla 1}

Instituciones estudiadas sobre marketing universitario e imagen institucional

UJAT IUP
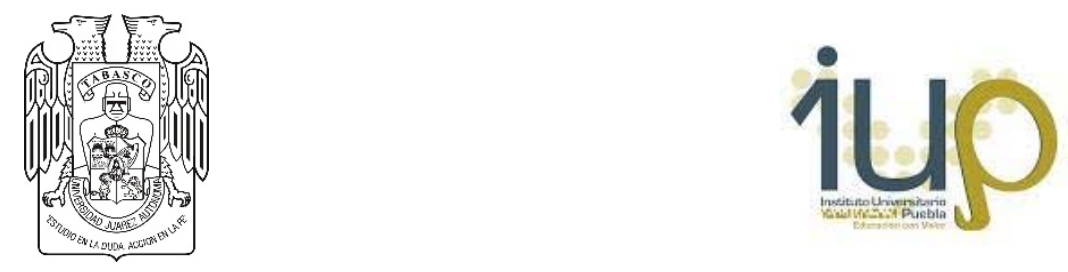

Fuente: UJAT e IUP.

En la UJAT se entrevistaron a 66 mujeres y 34 hombres, en un rango de edad entre los 18 y 34 años de edad. Con respecto al IUP, los informantes fueron 58 mujeres y 42 hombres en un rango de edad entre los 19 y 45 años de edad.

El diseño del instrumento privilegió solo tres dimensiones del marketing universitario: imagen institucional, servicios educativos y percepción de los competidores (ver tabla 2). 
MARKETING DE UNIVERSIDADES, ¿OFERTA EDUCATIVA O IMAGEN?

Tabla 2

Dimensiones estudiadas del marketing universitario

\begin{tabular}{ll}
\hline Dimensión & \multicolumn{1}{c}{ Definición } \\
\hline $\begin{array}{l}\text { Imagen } \\
\text { institucional }\end{array}$ & $\begin{array}{l}\text { Este factor se refiere a la percepción de los estudiantes acerca de la } \\
\text { imagen de su institución. } \\
\text { Servicios }\end{array}$ \\
$\begin{array}{l}\text { Esta dimensión intenta conocer la oferta de servicios que los estudiantes y } \\
\text { profesores conocen de su universidad. }\end{array}$ \\
$\begin{array}{l}\text { Imagen } \\
\text { corporativa de }\end{array}$ & $\begin{array}{l}\text { Esta dimensión está relacionada con la valoración de los estudiantes y } \\
\text { profesores respecto a la mejor universidad competidora de su institución. }\end{array}$ \\
\hline
\end{tabular}

Fuente: Camacho, Ancona y García-Muñoz (2010).

Con tales dimensiones se estructuraron las tres preguntas dirigidas a los estudiantes, las cuales fueron muy precisas queriendo confirmar la relevancia de la imagen institucional de las universidades estudiadas, como resultado del marketing universitario que estas organizaciones realizan (ver tabla 3).

\section{Tabla 3}

Preguntas del sondeo flash dirigido a jóvenes universitarios

\begin{tabular}{ll}
\hline \multicolumn{2}{c}{ Pregunta } \\
\hline 1. ¿Qué factor considera el más & a. Imagen institucional \\
importante de la universidad & b. Plan estratégico \\
donde presta sus servicios & c. Servicios educativos \\
profesionales? & d. Precio \\
& e. Internacionalización \\
& f. Prestigio \\
& g. Antigüedad
\end{tabular}


MARKETING DE UNIVERSIDADES, ¿OFERTA EDUCATIVA O IMAGEN?

2. La imagen de su universidad, la consideras.
a. Prestigiada
b. Vanguardista
c. Socialmente responsable
d. De Calidad
e. Integral
f. Con reconocimiento público
g. Acreditada internacionalmente

3. Comparado con su universidad ¿qué otra institución otorga mejores servicios educativos?
a. Universidad Autónoma de Guadalajara (UAG)
b. Tec Milenio
c. UJAT
d. Universidad Olmeca
e. Mundo Maya
f. Universidad de Sotavento
g. Alfa y Omega
h. Interamericana del Norte
i. Otra (especificar)

Fuente: Camacho et al (2010).

Las respuestas al sondeo flash, corresponden en la estructura del marketing universitario (Tristá, 2002), al posicionamiento (imagen institucional) y competitividad (de los productos de la universidad y de la competencia).

\section{Imagen institucional}

Los alumnos de la UJAT tienen dos importantes opiniones de su institución universitaria, y es que para ellos la imagen de su universidad está basada por el reconocimiento público, por su antigüedad y en menor proporción creen que la institución tiene prestigio y que es de calidad.

En un análisis comparativo básico de lo encontrado por Camacho et al (2010) se pudo apreciar el cambio de percepción de esta universidad (ver tablas 4 y 5). 
MARKETING DE UNIVERSIDADES, ¿OFERTA EDUCATIVA O IMAGEN?

Tabla 4

Factor determinante para la elección de los estudiantes por la UJAT

Año 2010 Año 2018

Imagen institucional Servicios educativos

Fuente: Elaboración propia con datos de Camacho et al (2010).

Estudiantes de la IUP, declararon que la imagen que brinda su universidad es de calidad y que además es socialmente responsable, algunos piensan que tiene reconocimiento público y una minoría cree que es prestigiosa y vanguardista.

\section{Tabla 5}

Factor determinante para seleccionar a la IUP

Año $2010 \quad$ Año 2018

Servicios educativos $\quad$ Plan estratégico

Fuente: Elaboración propia con datos de Camacho et al (2010).

\section{Servicios educativos}

Después de analizar los datos, se encontró que los estudiantes de la universidad pública eligieron a esta institución debido a su oferta educativa, este fue el principal motivo para ingresar a ella. Los alumnos de la universidad privada coincidieron que el factor más importante para optar por el ingreso a esta institución fue la oferta educativa de la misma. Aproximadamente el $80 \%$ de los alumnos a los que se les aplicó el instrumento, encontraron como principal atractivo los servicios educativos de ambas universidades.

\section{Imagen corporativa de los competidores}

Para los alumnos de la universidad pública, en su mayoría, opinaron que, comparado con su institución, la Universidad Olmeca y el Tec Milenio Universidad (universidades privadas) son mejores debido a la calidad de sus servicios educativos. En menor proporción mencionaron a la UAG. 
MARKETING DE UNIVERSIDADES, ¿OFERTA EDUCATIVA O IMAGEN?

Respecto a la opinión de los jóvenes de la universidad pública, afirmaron que existen otras instituciones que cuentan con mejores servicios educativos que su propia universidad, dieron como referencia a la UAG, UVM y Universidad OImeca (instituciones privadas).

\section{CONCLUSIONES}

El marketing universitario basado en el marketing estratégico, es fundamental para las IES no solo para promover su oferta educativa básica, sino también la complementaria (diplomados, cursos, consultoría, residencias, incubadoras de negocios, oficinas de transferencia de tecnología. Además, debe procurar el logro del posicionamiento de su imagen institucional en sus segmentos de mercado. En este proceso se deben difundir aspectos como la calidad, responsabilidad social, perfil de consumidores, competitividad de la planta docente, infraestructura y planes estratégicos por citar algunos componentes.

De acuerdo a los hallazgos de la investigación, se puede apreciar que cada vez más los estudiantes están atentos a la imagen institucional de sus universidades y la competitividad de estas frente a los competidores. Después de la observación participante y la aplicación del sondeo flash a 200 informantes seleccionados intencionalmente, se encontró que la percepción que los alumnos tienen de la imagen institucional de las universidades pública y privada que se estudiaron, se basan en los servicios educativos que estas ofrecen, además de los planes estratégicos y el prestigio que estas organizaciones posean.

Además, se puede decir que, para los alumnos de ambas universidades es de suma importancia la oferta de servicios educativos para la toma de decisiones en la selección de la IE en la que estudiarán su profesión. En este sentido, es relevante mencionar que para los alumnos de la UJAT es más importante la calidad educativa, comparado con la opinión de los alumnos de la IUP que basan su percepción en la oferta de servicios educativos, prestigio y reconocimiento público de una universidad.

Con estas perspectivas, se aprecia la oportunidad de que ambas universidades se apoyen en nuevas estrategias de marketing para posicionar sus buenas prácticas y la oferta diferenciada que tienen cada una. En este caso es conveniente utilizar el inbound marketing con acciones en social media para fortalecer la imagen institucional de las IES estudiadas. Los comunicados se difundirán a través de las principales redes sociales como: Facebook, Twitter e Instagram. 
MARKETING DE UNIVERSIDADES, ¿OFERTA EDUCATIVA O IMAGEN?

Se sugieren nuevos estudios sobre el uso y beneficios de las redes sociales para posicionar a universidades públicas o privadas. Con este trabajo se añade valor al estudio de la relevancia que tiene el marketing universitario en su vertiente de posicionamiento y competitividad en los mercados educativos.

\section{REFERENCIAS BIBLIOGRÁFICAS}

Arroba, J. (junio, 2000). ¿Cuándo y cómo se hace un sondeo flash? Revista latinoamericana de comunicación. Chasqui. Recuperado de: http://www.redalyc.org/pdf/160/16007007.pdf

Camacho Gómez, M. (2011). Nuevos retos internacionales para las universidades mexicanas. Perspectivas Docentes. Recuperado de: http://revistas.ujat.mx/index.php/perspectivas/article/viewFile/546/453

Camacho Gómez, M., Ancona Alcocer M. \& García-Muñoz, Aparicio, C. (2010). Mercadotecnia de servicios educativos. Trabajo presentado en XV Congreso Internacional de Investigación en Ciencias Administrativas de la Universidad Veracruzana en Boca del Rio, Veracruz, México.

Camacho Gómez, M. (2015). Business marketing services. A mexican approach. México. Universidad Juárez Autónoma de Tabasco.

Costa, J. (2009). Identidad corporativa. México: Trillas.

Doña, L. (2014). Marketing y universidad: análisis desde la perspectiva del egresado (Tesis Doctoral, Universidad de Granada). Recuperada de https://hera.ugr.es/tesisugr/24034277.pdf

De la Fuente Mella, H., Marzo Navarro, M. \& Reyes Riquelme, M.J (2010). Análisis de la satisfacción universitaria en la Facultad de Ingeniera de la Universidad de Talca. Ingeniare. Revista Chilena de ingeniería, 18(3), pp. 350-363.

Fayos, T., Gonzalez-Gallarza, M., Servera, D. y Arteaga, F. (2011). Análisis y evaluación del servicio de formación universitaria: implicaciones para el marketing estratégico de las universidades. Revista de Investigación en Educación, 9(2), pp. 133-152. 
MARKETING DE UNIVERSIDADES, ¿OFERTA EDUCATIVA O IMAGEN?

Instituto Universitario de Puebla. Recuperado de: http://www.iupuebla.edu.mx/

Loveclock, C. y Wirtz, J. (2009). Marketing de servicios. Personal, tecnología y estrategia. México: Pearson.

Martínez Miguelez, M. (2007). Ciencia y arte en la metodología cualitativa. México: Trillas

Tristá Pérez, B. (2002). Aproximación a un marketing universitario. Revista cubana de educación superior, 22(1), pp. 15-36.

Universidad Juárez Autónoma de Tabasco. Recuperado de http://www.ujat.mx

Wörner, C.H. y Santander, P. (2012). Marketing en la educación superior chilena. Recuperado de: http://www.observatoriodecomunicacion.cl/sitio/wp-content/uploads/2012/09/Marketingsistema-universitario.pdf 